Philosophie ANTIQUE

\section{Philosophie antique}

Problèmes, Renaissances, Usages

17 | 2017

Platon et la politique

\title{
Purifications et distributions sociales : Platon et le pastorat politique
}

\section{Arnaud Macé}

\section{(2) OpenEdition}

1 Journals

Édition électronique

URL : https://journals.openedition.org/philosant/285

DOI : 10.4000/philosant.285

ISSN : 2648-2789

Éditeur

Éditions Vrin

\section{Édition imprimée}

Date de publication : 1 novembre 2017

Pagination : 101-123

ISBN : 978-2-7574-1807-9

ISSN : $1634-4561$

\section{Référence électronique}

Arnaud Macé, «Purifications et distributions sociales : Platon et le pastorat politique », Philosophie antique [En ligne], 17 | 2017, mis en ligne le 01 novembre 2018, consulté le 03 décembre 2022. URL http://journals.openedition.org/philosant/285; DOI : https://doi.org/10.4000/philosant.285

\section{(c) $(1) \&$}

Creative Commons - Attribution - Pas d'Utilisation Commerciale - Pas de Modification 4.0 International - CC BY-NC-ND 4.0

https://creativecommons.org/licenses/by-nc-nd/4.0/ 


\section{PURIFICATIONS ET DISTRIBUTIONS SOCIALES : PLATON ET LE PASTORAT POLITIQUE}

Arnaud MACÉ

Université de Bourgogne Franche-Comté / Logiques de l'Agir EA 2274

amace@univ-fcomte.fr

RÉSUMÉ. Le présent article propose de réévaluer le rôle de l'analogie entre l'art politique et l'art pastoral dans la philosophie politique de Platon et d'offrir ainsi à l'histoire des arts de gouverner un autre aspect du pastorat que celui que Michel Foucault a cherché hors du monde grec. On a négligé de lire dans cette perspective le passage des Lois, V, 734e-736b où Platon invoque le gardien du troupeau pour mettre en avant une dimension fondamentale de l'art politique, celle qui consiste à purifier le corps social de ses éléments indésirables, mais aussi celle qui exige de subdiviser celui-ci pour mieux répartir en son sein les ressources et développer ses capacités d'action. On montrera comment Platon, en réactivant l'analogie homérique entre pratiques pastorales et pratiques militaires, dessine un art de gouverner inspiré par l'art de trier et de répartir des pasteurs d'hommes, comme un art de production de communautés robustes dotées d'une capacité d'agir collective.

SUMMARY. This paper aims at reassessing the role of the analogy between the political art and the art of the shepherds within Plato's political philosophy and thus at providing the history of the arts of governing with another aspect of the pastorate than the one Michel Foucault found outside of the Greek world. One has neglected to read in this light a passage of Plato's Laws, V, 734e-736b, where Plato invokes the art of the shepherds to highlight a fundamental dimension of the political art, that is the "purifications" performed to cast away unwanted elements from the social body, but also the subdivisions of the social body created to distribute goods among its members and enhance its capacity of action. In doing so, Plato reactivates homeric analogies between the art of shepherds and the art of military chiefs, and designs the art of governing after the art of subdividing and distributing displayed by the shepherds of men, as an art of producing robust communities endowed with a collective capacity for action.

Philosophie antique, ${ }^{\circ} 17$ (2017), 101-123 

Platon ${ }^{1}$, dans un passage peu commenté mais crucial des Lois, a donné à l'analogie pastorale une dimension fondamentale pour penser l'art politique, à tel point qu'elle invite à relire différemment l'usage de cette analogie à travers les dialogues, aussi bien qu'à mesurer les nouvelles résonances qui s'établissent alors entre la figure platonicienne du berger et celle qui se dessine dans les textes homériques. Nous voudrions donner toute sa portée à un fait qui est de nature à modifier la représentation que l'on peut être tenté de se faire de la présence de ce motif de la pensée politique des $\mathrm{Grecs}^{2}$, comme de la place de celle-ci dans l'histoire de l'usage de l'analogie pastorale dans la pensée politique en général. Cette histoire plus vaste est celle dont Michel Foucault avait entrepris d'esquisser les linéaments, en faisant l'hypothèse d'une séparation entre le « pasteur hébraïque » et le « magistrat grec », qui pourrait servir à établir que « la réflexion grecque sur la politique est exclusive de cette valorisation du thème du berger », telle qu'on la trouve « chez les Orientaux et chez les Hébreux $\gg^{3}$. La restitution du pastorat politique platonicien et son articulation avec l'art homérique des pasteurs d'hommes, dont on a bien établi l'application principalement militaire ${ }^{4}$, bouscule en effet, comme nous allons le voir, les digues que Foucault, dans un examen préliminaire des sources 5 , avait tenté de dresser pour isoler les affleurements

1. Je tiens à remercier chaleureusement le relecteur ou la relectrice anonyme qui m'a permis d'enrichir la mise en perspective de l'ensemble de l'argumentation et l'a nourrie de très heureuses suggestions en bien des endroits. J'espère qu'il ou elle lira ces lignes et que nous pourrons prolonger cette discussion.

2. Sur ce terrain, voir les références collectées par Ruby Blondell (Blondell 2005 p. 23-26), par Roger Brock (Brock 2013, p. 43-52) et par Johannes Haubold (Haubold 2000 p. 17-46).

3. Foucault 2004, p. 150.

4. Les études de R. Brock et J. Haubold, précédemment citées, sont très claires sur ce point. Voir par exemple Brock 2013 p. 43.

5. Foucault insistait sur le caractère hypothétique de ce qu' il esquissait alors : « Tout ceci, ces réflexions sur la gouvernementalité, cette très vague esquisse du pastorat, ne prenez pas ça pour argent comptant, bien entendu. Ce n'est pas du travail achevé, ce n'est même pas du travail fait, c'est du travail en train de se faire. » (Foucault 2004 p. 139). 
du thème pastoral dans la pensée grecque et en contenir l'influence au sein de ce champ culturel. Ce faisant, cette restitution offre aussi de nouvelles possibilités à l'histoire de la « gouvernementalité », au sens d'une histoire qui aborde la question des institutions et de l'État à partir de celle des techniques et des savoirs qui les irriguent et déterminent les formes multiples de pouvoir dont une population peut faire l'objet.

Échappant au pastorat politique, le monde grec et le monde romain offraient à Foucault un champ d'études apaisé des techniques de soi ${ }^{6}$, sans affecter par ailleurs la représentation d'un pastorat réduit à sa figure orientale, hébraïque et chrétienne, une figure dans laquelle la bienveillance et la disponibilité du berger à l'endroit de toutes et de chacune de ses brebis préparaient les formes individuelles d'assujettissement dont on pourrait lire le déploiement dans les savoirs et les institutions modernes. Or, la figure pastorale que nous nous apprêtons à restituer remet en cause de tels partages, notamment parce qu'elle fait paraître un autre visage du berger : son rôle de purificateur du troupeau, d'éradicateur des natures qu'aucune technique de soi ne saurait disposer à faire partie d'une communauté saine. Mais c'est encore par un autre trait que le pastorat politique platonicien vient perturber ces partages, en réclamant sa place au cœur du politique : le pasteur platonicien ne s'en tient pas à l'épuration qui précède l'institution proprement politique, il transpose l'art homérique de tri et de répartition des individus dans la troupe armée pour proposer une méthode de division et de ventilation du corps social qui confère à la cité platonicienne sa forme politique et sa capacité d'action. Par là, il révèle à quel point la Grèce ancienne, à condition de ne pas l'exclure d'une telle histoire, est un laboratoire de la « gouvernementalité » à ciel ouvert, tant la question du pouvoir y est constamment soumise à celle de la diversité des arts et des techniques à partir desquels il peut être pensé.

\section{Foucault et le pastorat politique des Grecs : une stratégie multiforme de contournement}

La stratégie de Foucault pour affirmer que la pensée politique des Grecs est « exclusive » de la valorisation du thème pastoral, dont témoignent à ses yeux les cultures du Proche-Orient, est multiforme ${ }^{7}$. Il s'agit d'abord de cantonner les champs où une certaine valorisation du modèle pastoral apparaîtrait dans la culture grecque, afin de pouvoir leur appliquer des traitements

6. Foucault 2001.

7. Ces thèses sont d'abord présentées sous la forme encore expérimentale, à la recherche d'elle-même, du cours prononcé au Collège de France le 15 février 1978 (Foucault 2004, p. 139-165), avant d'être reprises devant le public de Stanford dans le cadre des conférences prononcées les 10 et 16 octobre 1979 (Foucault 1981) et dont une traduction française est parue à titre posthume en 1986 (Foucault 1986), avant d'être reprise dans les Dits et Écrits (Foucault 1994 p. 134-161). 
différents de nature à en limiter la portée. Foucault accepte de reconnaître trois principaux foyers au sein de cette culture : l'image des « pasteurs d'hommes » appliquée aux rois homériques, certains textes pythagoriciens ou pseudo-pythagoriciens, et certains textes de la prose classique, dont Platon. Les deux premiers foyers sont neutralisés par des opérations opposées ${ }^{8}$ : l'appellation de « berger des peuples » serait un lieu commun « dans toute la littérature indo-européenne » sans portée politique véritable ${ }^{9}$; l'usage de l'image du magistrat bienveillant, du berger royal, signalerait les influences orientales à l'œuvre au sein des communautés pythagoriciennes, et confirmerait qu'il s'agit là d'une soumission du politique à des modes de gouvernement qui correspondent mieux à des communautés destinées à la progression épistémologique et morale d'un petit groupe d'individus, qu'à l'expérience politique de la cité ${ }^{10}$. Le corpus classique suppose à lui seul de multiples stratégies. La première est de constater la rareté du thème chez les auteurs supposés restituer davantage que les autres la vie politique de la Grèce classique (Isocrate, Démosthène, Aristote) ${ }^{11}$. Enfin Platon, chez qui l'importance du thème ne peut être niée, exige à lui seul un traitement multiple : il faut d'un côté renvoyer les textes où le paradigme semble valorisé pour penser l'art politique au filon pythagoricien dont Platon serait

8. Foucault 2004, p. 140-142.

9. Foucault tire peut-être cette affirmation de la lecture du chapitre où Benveniste discute cette expression (Benveniste 1969, p. 89-95). On se reportera néanmoins à J. Haubold et aux références qu'il rassemble sur le fait, d'une part, que la métaphore n'est pas un lieu commun mais permet une analogie vivante - nous reviendrons sur cet aspect - et, d'autre part, que l'expression est répandue dans les cultures non «indo-européennes » de Mésopotamie et du Proche-Orient et qu'on a des raisons de penser que l'usage qu'en font les Grecs s'en inspire (Haubold 2000 p. 17, en particulier n. 3 et 5).

10. Alain Petit a développé une lecture du pastorat dans la pensée pythagoricienne (en particulier à partir des témoignages sur la pensée d'Aristoxène de Tarente et du traité pseudépigraphique Sur la Royauté) inspirée de thèses foucaldiennes, en confirmant la vocation du pastorat à « rethéologiser »l'exercice du pouvoir (Petit 1999).

11. Isocrate et Démosthène sont cités dans le cours (Foucault 2004 p. 142), Aristote les rejoint dans les conférences de Stanford (Foucault 1981 p. 141). Michel Sénellart, dans une note au passage pertinent dans le cours, a ajouté un exemple, qui permettrait de déminer la présence du motif chez des auteurs classiques en affirmant qu'il n'apparaît qu'en parlant de populations étrangères, ainsi chez Xénophon à propos des Perses, Cyropédie, I, 1, 1-3 et VIII, 2, 14 (Foucault, 2004, note 10 p. 161). D. El Murr a noté que l'argument pourrait être étendu à Platon lorsque celui-ci aborde le régime de Cyrus dans les Lois (III, 694e-695a), tout en remarquant judicieusement que le fait même que « Xénophon et Platon notent tous deux l'origine perse du modèle pastoral » n'implique pas pour autant que « ce modèle n'ait aucune importance ni aucun rôle positif à jouer dans leur pensée politique » (El Murr 2013 p. 185). Il n'est pas sûr que ces passages signifient que Xénophon et Platon voient dans la Perse l'origine du modèle, mais il est certain que le modèle de Cyrus est loin d'être simplement repoussé et qu'il fait au moins l'objet d'une réappropriation complexe de la part des deux auteurs (Azoulay 2004 p. 197-203 ; Pontier 2006 p. 404-408). 
héritier ${ }^{12}$, réduire en outre cette reconnaissance à des formes subalternes de magistrature $^{13}$, et surtout neutraliser l'usage du modèle dans le Politique en y voyant une critique en règle par Platon d'une analogie qu'il abandonne aux pythagoriciens, comme une forme subalterne d'activité politique désormais soumise à un art véritablement politique, celui du tissage ${ }^{14}$. Dimitri El Murr s'est récemment opposé à cette lecture du Politique, en montrant notamment que le paradigme du tissage s'appuie sur le pastorat davantage qu'il ne le remet en cause, pour en prolonger la dimension d'art de $\operatorname{soin}^{15}$ : le fait que le pastorat du temps de Cronos suppose que les hommes aient pour bergers des dieux n'implique pas que le tissage de la cité des hommes par les hommes cesse de chercher à imiter en quelque façon le pastorat perdu ${ }^{16}$. Le fait que

12. Cette stratégie est employée à propos de la suggestion par Thrasymaque que les gouvernants se rapportent à leurs gouvernés comme les pasteurs et bouviers à leurs troupeaux ou leurs bœufs (République, I, 343a-c). À vrai dire, Foucault semble d'abord vouloir opter pour la stratégie qui consisterait à voir dans l'argumentation de Thrasymaque une récusation du modèle du berger pour penser l'autorité politique : «Donc, dit Thrasymaque, cette comparaison avec le berger n'est absolument pas topique pour caractériser la vertu nécessaire au magistrat. »(Foucault 2004 p. 143). L'argument semble s'appuyer sur l'idée que la proposition par Thrasymaque d'un berger qui ne s'occupe des brebis que pour son profit devrait être une critique interne du modèle - mais cela ne fonctionne que si l'on a décidé que le pouvoir pastoral se fondait sur l'affirmation d'une bienveillance (un des traits que Foucault définit au cours précédent, voir p. 130 : « le pouvoir pastoral est, je crois, tout entier défini par sa bienfaisance »), ce qui ne va pas de soi, comme nous allons le voir, en tout cas chez Platon. Ensuite, face à la réponse de Socrate, qui valide l'analogie entre pouvoir politique et berger, pour peu qu'on comprenne bien le berger comme celui qui agit dans l'intérêt de son troupeau (345b-347a), Foucault se résout à marginaliser Platon : « c'est ce thème pythagoricien du magistrat-berger, de la politique comme bergerie, c'est ce thème-là qui affleure, je crois, clairement dans le texte de la République, au livre I » (p. 144). Si tel est le cas, ce n'est pas seulement le livre I qu'il va falloir relativiser. Comme l'a indiqué Dimitri El Murr, l'analogie pastorale est un motif qui revient dans la construction de la cité idéale, notamment avec la mise en place de la triade berger - chien de garde - troupeau (El Murr 2013 p. 175-177).

13. C'est la stratégie adoptée à propos de l'usage de l'analogie pastorale pour décrire la façon dont les dieux administrent le progrès moral de l'humanité, au livre X des Lois (Foucault 2004 p. 143-144). Nous allons revenir sur ce passage et sur le fait que Foucault ne prend pas bien la mesure de la dimension hiérarchique du schème pastoral chez Platon : le fait que le berger ait des aides (typiquement le chien, mais pas seulement) ne fait pas descendre la fonction pastorale au niveau subalterne.

14. Dimitri El Murr rappelle qu'il s'agit au fond d'une reformulation d'une thèse présente dans la littérature (voir par exemple la traduction de J. B. Skemp en 1952) selon laquelle le Politique critiquerait la forme monarchique du pouvoir telle qu'elle apparaît dans la Cyropédie de Xénophon. D. El Murr cite la variante dont M. Narcy a fourni une récente version (Narcy 1995) : la cible de l'Étranger est la philosophie politique de Socrate, telle qu'elle peut être illustrée par le modèle pastoral de la République (El Murr 2013 n. 2 p. 177).

15. El Murr 2013, p. 183-195.

16. Alain Petit (Petit 1999 p. 11-12) rappelait déjà une remarque d'Henri Joly (Joly 1994 p. 290), sur le fait que dans le Politique le pastorat du mythe reste un modèle « disponible » pour l'art de gouverner du temps de Zeus. 
d'autres figures que le pasteur puissent relayer celui-ci dans un dispositif platonicien fragilise aussi certains arguments de Foucault sur d'autres auteurs, ainsi particulièrement à propos d'Isocrate. Foucault tient en effet à mobiliser l'Aréopagitique où il considère que la description des devoirs du magistrat, qui inclut celui de prendre soin des jeunes gens, de les surveiller sans cesse, de veiller aussi bien à leur nourriture qu'à leurs mœurs, nous amène « tout près de la métaphore du berger $\gg$, sans pour autant que celle-ci intervienne ${ }^{17}$. L'argumentation pourrait s'avérer volatile : montrer à quel point les traits du pastorat politique peuvent se retrouver diffusés dans les œuvres de l'époque classique sans qu'il soit besoin d'en mobiliser littéralement la figure pourrait aussi servir à prouver que ce thème est si prégnant que ses traits sont susceptibles de se déployer librement, sans que la figure en soit nommément invoquée, ou de se transmettre à d'autres figures connexes.

\section{L'art pastoral avant le tissage : le pastorat purificateur (Lois V et Politique)}

Il y a au moins un lieu important que Foucault a omis de relever chez Platon, à l'un des moments les plus cruciaux de la fondation de la cité des Lois $^{18}$. Il faut commencer par ce simple fait, qui jette une lumière nouvelle sur l'ensemble des sources. Au livre V, on commence par énoncer un préambule à la constitution, qui appelle chacun à honorer son âme, en cultivant en elle les vertus, et s'achève sur le calcul des plaisirs qui établit que la vie vertueuse est plus plaisante que la vie vicieuse. Il faut alors passer au morceau principal :

Voilà formulé le préambule que nous voulions donner à notre législation, et c'est ici qu'il doit atteindre son terme. Et après ce préambule, il faut que vienne un air, ou à vrai dire il faut plutôt tracer une esquisse des lois de la constitution (vó situation que si nous fabriquions un tissu ou tout autre objet tressé ( $\tau$ iv $\alpha$

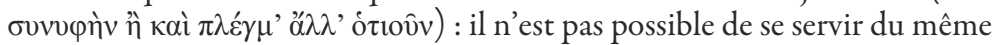

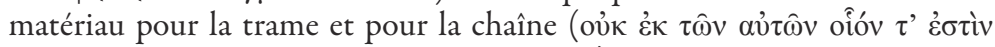

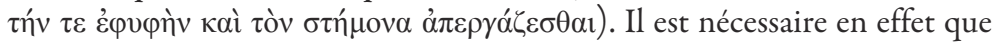
la chaîne soit d'une qualité supérieure - car elle a pour qualité la force et une certaine solidité -, tandis que la trame est plus molle et exige une juste souplesse. De là vient donc que c'est un peu de la même façon que doivent être départagés respectivement et proportionnellement les magistrats qui auront la direction de la cité et ceux dont la qualité aura été éprouvée par

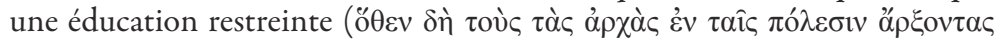

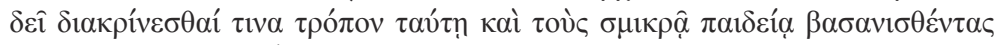

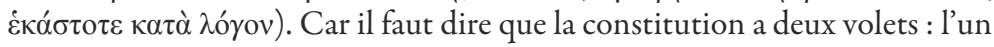

17. Foucault 2004 p. 142.

18. Le passage échappe aussi à Roger Brock, qui pourtant suit le motif jusque dans les Lois, à propos de Cyrus, en 694c et 695a (Brock 2013 p. 48). 
est l'attribution des magistratures aux individus, l'autre est l'institution des lois pour ces magistrats ${ }^{19}$.

La reprise du motif du tissage, déjà utilisé dans le Politique, est très nette, avec l'idée, toujours la même, que la trame réclame un matériau plus solide et que c'est la souplesse qui fera la qualité de la chaîne ${ }^{20}$. Avant de poursuivre sur le sujet des magistratures et de clarifier en quoi l'attribution de celles-ci et l'institution des lois correspondraient respectivement à l'établissement de la trame et de la chaîne, l'Étranger interrompt l'analogie pour en introduire une autre, dont l'invocation est motivée par un problème semble-t-il plus urgent :

Mais il faut avant tout prendre en considération ce que voici. Quiconque a reçu la garde d'un troupeau, berger, bouvier, éleveur de chevaux ou toute

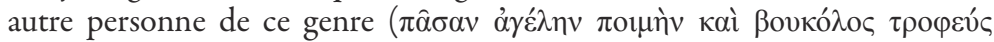

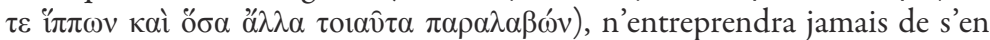
occuper ( $\theta \varepsilon \rho \alpha \pi \varepsilon v \varepsilon \varepsilon v)$ avant d'avoir purifié chaque troupeau par l'espèce de

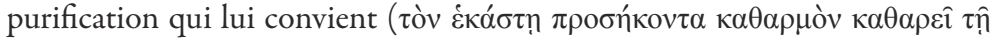

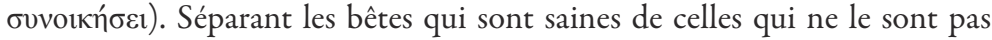

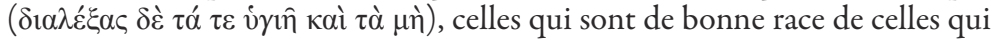
ne le sont pas, il renverra les secondes dans d'autres troupeaux et il s'occupera des premières, en considérant qu'il serait vain et inutile de faire des efforts pour un corps et pour des âmes que leur naturel et un mauvais élevage ont corrompus et qui en outre ruinent ce qu'il y a de sain et de bon dans les mœurs comme dans les corps de chacune des bêtes du troupeau, et qu' il serait fatal de ne pas purifier le troupeau dont il a présentement la garde ${ }^{21}$.

Voilà une description du rôle des pasteurs et bouviers qui n'est pas toujours portée au premier plan, et il faut saluer la façon dont Vincent Azoulay l'a récemment mise en valeur ${ }^{22}$. La purification du troupeau, c'est pour ainsi dire l'inverse du tissage : au lieu de tenter d'accorder des naturels opposés, il s'agit ici de repérer et d'évacuer les naturels susceptibles de contaminer le troupeau, qu'il s'agisse de maladies ou de mauvais comportements - il s'agit bien de trier les corps et les âmes, en isolant les maladies et les comportements déviants ${ }^{23}$. Cette conception de la purification s'intègre

19. Platon, Lois, V, 734e3-735a6. Sauf indication expresse, nous citons toujours la traduction Brisson \& Pradeau 2008.

20. Voir Politique, 308e-309b, nous y revenons ci-après. Dimitri El Murr annonce, au terme de son étude sur le Politique, que le modèle du tissage de la cité qu'il vient de décrire sera bien repris dans ce passage des Lois pour présider à la répartition des magistratures (El Murr 2014 p. 292 note 1$)$.

21. Platon, Lois, 735 a $7-c 3$.

22. Azoulay 2014 p. 692-697.

23. Il faudrait mentionner à quel point les opérations préalables au tissage, le lavage de la laine et le cardage de celle-ci, peuvent jouer le même rôle structurel pour signifier l'éviction des éléments qui ne pourront pas entrer dans le tissage, ainsi qu'en témoigne le passage de 
très bien à l'analyse qui en a été faite dans le Sophiste. La purification y est

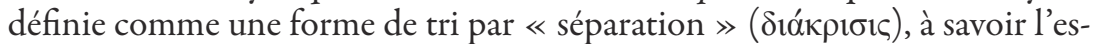

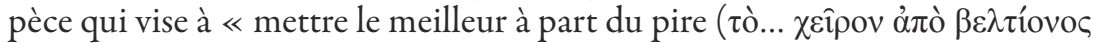

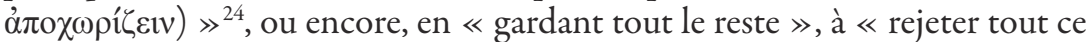

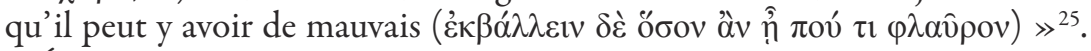
L'Étranger affirme que tous appellent une telle séparation une « purification $\gg(\kappa \alpha \theta \alpha \rho \mu o ́ c)^{26}$. On distinguera alors les purifications relatives au corps, qu'il soit vivant (purifications internes comme celles qu'opèrent la gymnastique et la médecine, ou externes comme celles que procure l'art du bain) ou inerte (le cardage, le foulage ${ }^{27}$, de celles qui ont l'âme pour objet, afin de débarrasser celle-ci de ses deux maux, analogues à la maladie et à la laideur

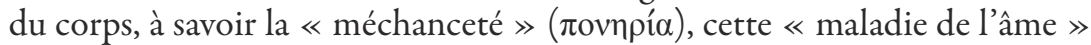

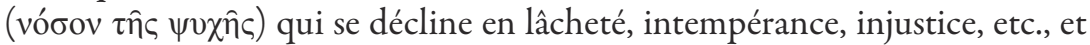
l'« ignorance » ('́xyor $\alpha$ ), qui en est la laideur, la disgrâce ( $\alpha \hat{\imath} \sigma \chi \circ \varsigma)$. L'équivalent de la médecine et de la gymnastique seront pour l'âme la justice et l'enseignement ${ }^{28}$.

Le texte des Lois nous propose par conséquent d'ajouter une nouvelle forme à cet art de la purification. Les pasteurs et bouviers n'ont pas été nommés dans le contexte du Sophiste, et il semble que l'on puisse comprendre pourquoi en considérant que la définition de la purification, si elle reste identique (trier le pire pour garder le meilleur), doit être dans leur cas raffinée en deux points : il ne s'agit pas de traiter des corps ou des âmes individuelles, mais un groupe dont on écarte les mauvais éléments; il s'agit de traiter dans la même opération aussi bien les corps que les âmes, en ciblant les corps malades et les âmes déviantes. On retrouve ici la dimension intégrale du pastorat : le berger est, pour son troupeau, aussi bien le médecin que l'éducateur. C'est un trait sur lequel l'Étranger insiste dans le Politique : le bouvier, comme tous les autres pasteurs, est, pour son troupeau, à la fois le nourricier, le médecin, le marieur, la sage-femme, l'éducateur qui fait découvrir les jeux et la musique ${ }^{29}$. C'est du reste, comme on l'a dûment noté ${ }^{30}$, ce qui fait surgir une difficulté pour transposer le modèle pastoral au corps politique, puisque dans ce cas le roi-berger sera assailli d'autant de prétendants qui

Lysistrata d'Aristophane (v. 574-586) qui précède le Politique de Platon dans l'usage politique du modèle du tissage - voir sur ce point Azoulay 2014 p. 695-696. Sur la comparaison du Politique de Platon et de la pièce d'Aristophane de manière plus générale, voir El Murr 2002.

24. Platon, Sophiste, 226d1-2.

25. Sph. 227d6-7.

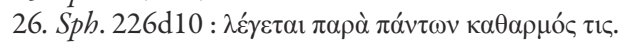

27. Sph. 226e5-227a5. Sur le cardage comme purification, voir le passage de Lysistrata précédemment cité note 23.

28. Sph. 227c7-229a10.

29. Platon, Politique, $268 \mathrm{a}-\mathrm{b}$.

30. Foucault 2004 p. 146-147. 
pourront justement prétendre accomplir mieux que lui chacune de ses tâches ${ }^{31}$. Notons simplement que l'objection des prétendants ne signifie pas nécessairement que l'art pastoral ne peut être l'art politique, mais seulement que le berger du troupeau humain devrait enrôler à son service un certain nombre de techniciens capables d'accomplir sous son égide la diversité des tâches que le pasteur accomplit seul avec ses chèvres - nous allons revenir sur cette suggestion. Le pasteur des troupeaux non humains est quant à lui tous ces spécialistes à la fois, et c'est bien ce qui lui permet d'identifier les bêtes à écarter du troupeau au moment où il le reçoit. Voyons dans quelles conditions se fait la transposition de ce modèle au groupe humain. Revenons à la lecture du passage des Lois :

À coup sûr, lorsqu'il s'agit d'animaux, l'intérêt est moins sérieux et il ne

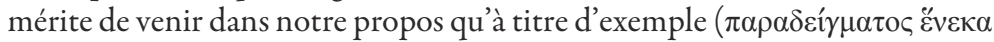
uóvov). Mais, lorsqu'il s'agit des hommes, il est du plus sérieux intérêt pour le législateur de rechercher et d'exposer avec soin ce qu'il convient de faire en chaque cas pour cette purification comme pour les autres mesures à prendre. Ainsi, en effet, pour ce qui a trait aux purifications à faire dans la cité, voici comment on pourrait procéder ${ }^{32}$.

L'exemple pastoral n'est qu'un exemple, mais qui n'attend qu'une transposition pour devenir un modèle pour les purifications correspondantes, celles de la cité ( $\pi \varepsilon \rho i ̀ ~ \kappa \alpha \theta \alpha \rho \mu o v ̀ \varsigma ~ \pi o ́ \lambda \varepsilon \omega \varsigma, 735 \mathrm{~d} 1$ ), dont il existe deux sortes, l'une rude et l'autre douce. Nous allons comprendre que le berger des bêtes ne nous avait encore montré que la face la plus douce des purifications pastorales :

Parmi les nombreux moyens de réaliser ces purifications, il y en a de plus doux et de plus sévères. Les plus sévères, qui sont aussi les meilleurs, c'est un législateur qui serait en même temps un tyran qui pourrait les appliquer. $\mathrm{Au}$ contraire, un législateur qui établirait une constitution nouvelle et instituerait des lois sans être un tyran, s'il procédait à la purification la plus douce, devrait se réjouir de seulement y parvenir. Or la purification la meilleure est douloureuse, tout comme le sont les remèdes efficaces. C'est celle qui conduit à la correction du coupable au moyen d'un châtiment accompagné d'une vindicte, et qui n'a que la mort ou l'exil comme vindicte dernière : ceux en effet qui ont commis les fautes les plus graves, comme ils sont incurables et constituent pour la cité le dommage le plus grand, il est d'usage de s'en débarrasser. Quant à la plus douce de nos deux purifications, la voici : à tous ceux qui, en raison de leur indigence, se révèlent être prêts, eux qui n'ont rien, à suivre leurs meneurs pour marcher en armes contre les biens de ceux qui en possèdent, à ces gens-là, comme à une maladie connaturelle à

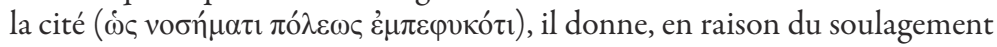

31. Plt. 267e-268a.

32. Lois, V, $735 \mathrm{c} 3-\mathrm{d} 1$. 
que procure l'euphémisme, le nom de « colonie » et il s'en débarrasse avec le plus de bienveillance possible ${ }^{33}$.

Le pastorat politique a deux moyens à sa disposition lors de l'établissement d'une constitution nouvelle. Le meilleur de ces moyens exige un pouvoir fort, et vise à l'éradication des éléments corrupteurs, par la mort ou l'exil. Le second des deux moyens sera celui que l'on utilisera face à un problème différent : la présence d'une masse de citoyens sans fortune, risquant de menacer les propriétés des riches. Il semble qu'il y ait deux degrés de maladie ici : celle, incurable, qui mène à la mort - on pense aux impies du livre $\mathrm{X}$ par exemple -, et celle que constitue la présence des pauvres. La description de la pauvreté comme maladie naturelle évoque la façon dont la pléonexie, au livre $\mathrm{X}$, sera à son tour décrite comme un phénomène général, qui « est appelé maladie dans les corps de chair, peste dans les saisons et les années, et dans les cités et les constitutions, par une modification du nom, injustice ( $\dot{\varepsilon} v$

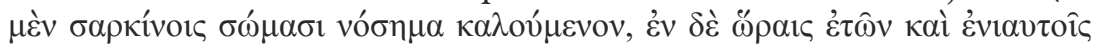

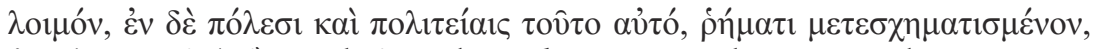

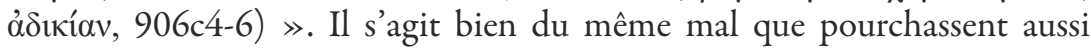
bien le médecin, le berger qui s'occupe des brebis, ou le fondateur de cité.

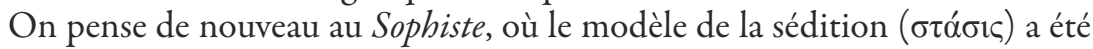
utilisé pour favoriser la transposition de la maladie du corps à celle de l'âme : il faut comprendre que dans l'âme des méchants les opinions et les désirs, les vertus, les plaisirs et les peines sont en désaccord les uns avec les autres, pour saisir la parenté originelle de la maladie et de la discorde ${ }^{34}$. Or ce que prévoit le législateur-pasteur, c'est précisément le développement d'une telle discorde du fait de la présence dans la cité d'un différentiel entre pauvres et possédants, qui rendra les premiers exposés aux tentations de mettre la main sur les richesses des seconds. Cette discorde sera la maladie du corps social, dont le pasteur est donc ici le médecin préventif ${ }^{35}$.

Pourquoi la seconde purification est-elle plus douce ? L'exil, envisagé avec la peine de mort comme moyen d'une purification plus dure, est une privation des droits civiques, interdisant à celui qu'elle frappe de reparaitre sur le sol de sa cité. En revanche, participer à la fondation d'une nouvelle colonie, c'est devenir citoyen d'une nouvelle cité qui conservera des liens privilégiés avec sa cité-mère. L'existence de ce « doux » moyen de purification implique en outre que la maladie que représentent les pauvres n'est pas incurable : elle est pour ainsi dire une maladie structurelle, car il suffit, semble-t-il, pour la faire cesser de faire cesser le différentiel qui en est la cause, en créant une nouvelle cité où les différences de fortune seront maîtrisées. En un sens, les

33. Lois, V, 735d1-736a3.

34. Platon, Sophiste, 228a4-b10.

35. Sur la transposition de la maladie à la cité, voir Macé 2010. 
riches, par leur présence ou par leur enrichissement excessif, sont autant responsables de cette maladie que les pauvres.

L'analogie se transforme finalement en métaphore hydraulique, qui correspond mieux au cas de la cité des Magnètes où il ne s'agit pas de purification - les colons seront choisis, ce ne sont pas des citoyens dont d'autres cités devraient se débarrasser. On les comparera plutôt à de multiples sources et de multiples torrents qui se déversent dans le même lac : il faudra seulement veiller à détourner certains affluents pour que l'eau soit la plus pure possible (736ab). Cela signifie que l'on pourra écarter certains candidats par une épreuve initiale (736c). Avec une population saine, le tissage va pouvoir commencer. Le pasteur, par un procédé de purification, donne au tisserand sa population.

Confirmons ce résultat en revenant vers le Politique, pour y relever un détail quelque peu troublant, au moment où l'on conclut sur la mise en œuvre du tissage, dans le grand texte final consacré à ce thème.

Il me semble que la science royale, qui possède en propre l'autorité de présider, en fera de même à l'égard de tous ceux qui sont chargés d'éduquer et d'élever conformément à la loi : elle ne permettra aucun exercice dont l'exécution ne produit pas une disposition susceptible de faciliter la combinaison qui est sa tâche propre, et qui ne produit pas les mœurs qui conviennent à cette combinaison, mais elle ne recommandera d'enseigner au contraire que des exercices qui la favorisent. S'il se trouve des gens qui ne sont pas capables d'avoir part au courage et à la réflexion, ni à toutes les autres tendances à la vertu, et que leur nature mauvaise les pousse au contraire vers l'athéisme, la démesure et l'injustice, elle s'en débarrasse en les condamnant à mort, à l'exil et aux peines le plus infamantes.

Du moins, c'est bien un peu ce que l'on dit.

Ceux par ailleurs qui se vautrent dans l'ignorance et l'abjection, elle les met sous le joug de l'esclavage.

Rien de plus juste.

Parmi ceux qui restent maintenant, tous ceux dont les natures sont assez bien nées pour se prêter à l'éducation et [309b] qui sont susceptibles, avec l'aide de la technique, de se mêler les uns aux autres pour atteindre la vertu, ceux qui sont plutôt portés vers la fougue, le roi investi de cette technique estime que leur caractère rigide marque leur place en quelque sorte dans la chaîne, tandis que les autres, qui inclinent vers la modération, notre roi trouve en eux la souplesse et la mollesse qui, pour filer notre image, conviennent à la trame ; et leurs tendances étant opposées, il s'efforce de les lier ensemble et de les entrecroiser de la façon suivante ${ }^{36}$.

On a souligné à quel point ici le tissage présuppose la tâche subalterne du cardage et du filage, qui sélectionne le matériau apte à être filé, en soulignant par conséquent le rôle de l'éducation et des épreuves qu'elle institue 
afin de repérer les bons naturels ${ }^{37}$. Ce texte consacré à l'art royal qui croise la chaîne et la trame de la cité pour en composer le tissu, introduit un détail qui n'était pas apparu précédemment dans le même dialogue : la double éviction de ceux qui ne peuvent pas entrer dans le tissu du corps des citoyens, soit par la mort et l'exil, soit par une voie plus douce, en l'occurrence la réduction en esclavage $^{38}$. La distinction des deux peines, la sévère et la plus douce, évoque par avance les purifications des Lois, tandis que la distinction des pathologies, celle des vices incurables et celle de l'ignorance, rappelle les purifications du Sophiste. La comparaison des textes nous amène à constater que les prérogatives qui seront introduites par une analogie avec la purification pastorale dans les Lois apparaissent ici au moment où le Politique dresse le portrait final de l'art royal. Insistons sur ce point : le texte dit très clairement que c'est celui qui détient l'art royal de tisser qui prononce l'éviction. C'est donc la science royale qui a la responsabilité de rejeter ce qui ne peut être tissé. Par ailleurs, la comparaison avec le texte des Lois impose de reconnaître que l'éviction n'est pas ici un moment préalable à l'institution du corps social : elle est conçue comme un pouvoir continu de prononcer l'éviction de tous les éléments, qui, au fur et à mesure de la vie de la cité et du renouvellement des générations, seront reconnus comme incapables d'entrer dans le tissage social.

Le Politique servait à Foucault à affirmer que le pouvoir pastoral est au mieux rendu subalterne. Or la restitution d'une nouvelle figure du pasteur - son visage purificateur -, venue des Lois, mais s'introduisant aussi subrepticement dans le Politique, bouleverse la donne : ce pasteur-là ne pourrait être contenu ni dans la subordination - puisque ses prérogatives sont celles de la science royale - ni dans le moment initial et somme toute pré-politique où semblent, mais nous allons y revenir, le maintenir les purifications préalables des Lois. Il faut pour l'instant commencer par revenir sur le caractère inquiétant de cette nouvelle figure pastorale.

\section{Le tri et les bergers : la raison de l'analogie}

Le pasteur que nous venons de rencontrer est aux antipodes de celui que Michel Foucault a décrit, en particulier relativement à l'un de ses traits fondamentaux, la bienveillance qui fait préférer au berger de risquer la vie du troupeau pour sauver une seule brebis égarée ${ }^{39}$. Ce nouveau pasteur est au contraire celui qui ne perdrait pas une minute à suivre une brebis égarée ou déviante qui menacerait l'intégrité du troupeau : il est celui qui la tuerait de ses mains pour couper court à toute contagion au sein du troupeau. Cela

37. El Murr 2014 p. 272-277.

38. Vincent Azoulay a bien vu que le motif de la purification revenait dès que Platon invoque la figure du pastorat sur le terrain politique, et dans le Politique et dans les Lois (Azoulay 2014 p. 694 n. 21).

39. Foucault 2004 p. 130-131. 
n'empêche pas que le pasteur platonicien puisse aussi manifester une forme de bénévolence à l'endroit du troupeau qu'il sert et soigne, mais cela signifie que nous devrons requalifier ce versant positif en lui donnant pour fond l'œuvre de purification - qui nous apparaît pour l'instant préalable à celle de soin. Or un autre trait du berger foucaldien est mis en doute : la dimension de service indéfini ${ }^{40}$. Le pasteur des Lois ne sert pas de manière indéfinie : il joue un rôle déterminant dans la fondation de la communauté, et, même si le texte du Politique semble ouvrir la perspective inquiétante de son retour, on pourrait imaginer que son action purificatrice reste ponctuelle. Bref, tout se passe comme si Platon venait de nous faire apercevoir une espèce du pasteur que le pastorat oriental de Foucault nous avait fait manquer. Peut-être n'y a-t-il là qu'un trait évanescent sorti de l'imagination fertile de Platon et qui ne mérite pas de remettre en cause l'essence du pasteur telle qu'elle est apparue chez Foucault ?

Revenons sur la façon dont Platon a fait paraître dans le Sophiste son concept de purification, qu'il associera dans les Lois au pasteur. La purification n'est qu'une des espèces d'un art plus fondamental que nous avons

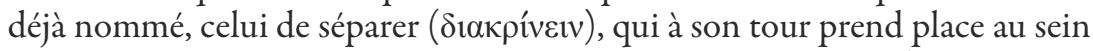
des opérations domestiques de « filtrer..., cribler, vanner, $\operatorname{trier}(\delta i \eta \theta \varepsilon i v . . . \kappa \alpha i$

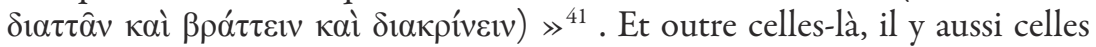

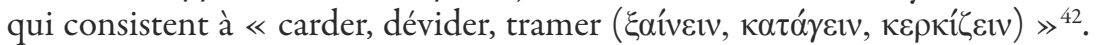
Or, dans tous les cas, il y a là un seul et même grand art, l'art « diacritique $(\delta ı \alpha \kappa \iota \tau \iota \kappa и ́) ~ \gg{ }^{43}$. Il est intéressant de se pencher sur l'histoire des usages du

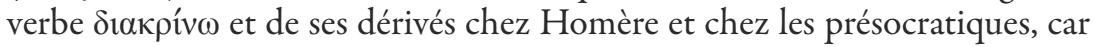
il apparaît que c'est la description de l'activité pastorale qui concentre chez

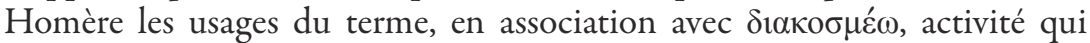
permet à Homère de décrire par analogie celle des « pasteurs d'hommes ${ }^{44}$. Le titre de « pasteurs d'hommes » est, chez Homère, non pas un simple lieu commun, mais le fruit d'une expérience de pensée analogique ${ }^{45}$, fondée sur l'observation d'une ressemblance procédurale entre l'action du berger et celle du chef militaire : on est un « pasteur d'hommes » parce que l'on fait

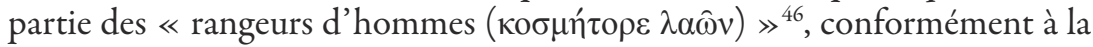
compétence la plus remarquable des pasteurs, celle de savoir trier les multitudes en les séparant :

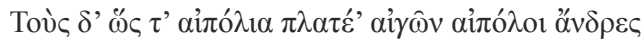

40. Foucault 2004 p. 131-132.

41. Platon, Sophiste, 226b5-6.

42. Sph. 226b8-9.

43. Sph. $226 c 8$.

44. Macé \& Therme 2013.

45. Sur cet aspect vivant de la comparaison, voir Haubold 2000 p. 17.

46. Homère, Iliade, III, 236. 


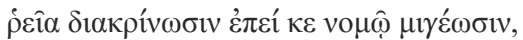

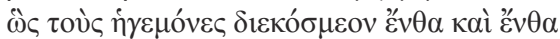

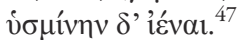

Comme les chevriers séparent facilement de vastes troupeaux de chèvres, lorsqu'ils se mélangent dans le pâturage, les chefs, ça et là, rangent, afin d'aller au combat.

L'analogie suggérée dans ces lignes se déploie en une structure remarquable, concentrique, où les rangeurs d'hommes « rangent» par leur présence même au centre d'un ensemble de rangeurs subalternes qui prolongent leur action : le roi est comme le berger qui guide l'ensemble des bêtes qui relaient son œuvre (chiens, mais aussi animaux dominants comme le bélier), il voit ses compagnons venir chacun à lui avec sa petite troupe et domine quant à lui l'ensemble des mouvements ${ }^{48}$. Sans la reconnaissance de cette structure, on peut être tenté, comme Michel Foucault, de penser que l'analogie pastorale du livre $\mathrm{X}$ des Lois accomplit l'assignation du rôle pastoral à un rang subalterne ${ }^{49}$. Lors de la troisième réfutation de l'impiété, celle des impies qui pensent que les dieux peuvent être corrompus, l'Étranger déploie une analogie d'abord militaire puis pastorale (905d3-907d1). Les dieux sont représentés comme des magistrats qui administrent le ciel, et sont comparés à ceux qui mènent des guerres : les conducteurs de chars, les pilotes de navires, ceux qui commandent à des armées $(\sigma \tau \rho \alpha \tau o \pi \varepsilon ́ \delta \omega v)$ ou même les médecins qui président à la guerre contre la maladie (905e7-10) ou l'agriculteur qui défend la plante contre le changement de saison. Les dieux, accompagnés des démons qui les assistent et des hommes qui sont leur propriété, mènent une vaste bataille pour faire triompher la vertu contre le vice (906a5-6). L'analogie guerrière est alors reformulée en termes pastoraux, et cela a pour effet immédiat de transformer la guerre en une guerre interne: mener la guerre devient protéger un troupeau contre ceux qui tentent de le corrompre. Cela amène une étrange vision du loup, devenu l'image de l'impie corrupteur, cherchant à corrompre « les âmes des gardiens du troupeau », « qu'il s'agisse de celles des chiens ou des bergers ou des maîtres les plus élevés de tous $\gg^{50}$, afin de laisser leur injustice impunie. Les loups, offrant une partie du butin aux chiens pour que ceux-ci les laissent dépecer le troupeau (906d2-4), sont devenus l'exemple même de la corruption intérieure, celle que la purification aurait dû faire disparaître. Or, comme on peut le montrer ${ }^{51}$, cette structure est mieux comprise si on rappelle la comparaison homérique de l'armée achéenne avec un ensemble de troupeaux : les chefs sont alors, selon leur rang, comparés à des bergers, à

47. Homère, Iliade, II, 474-477; cf. Allen 2000 [1931] (éd.). Nous traduisons.

48. Macé \& Therme 2013 p. 236-242.

49. Foucault 2004 p. 143-144.

50. Lois, X, 906b4-6.

51. Macé (à paraître-a). 
des chiens ou à des animaux dominants dans le troupeau, tout simplement parce que chacun de ceux-ci joue un rôle dans la remise en ordre du troupeau, chacun à son niveau, ainsi lorsque les chefs homériques qui officient sous le regard d'un roi sont comparés au bélier que le berger voit fièrement guider les autres bêtes (III, 196 et XIII, 492-493). Cette structure hiérarchique, loin de restreindre la fonction pastorale à l'échelon subalterne, la diffuse en réalité du haut en bas et de bas en haut, car c'est l'ensemble de la structure qui est rendue nécessaire pour accomplir la tâche pastorale : le maître regarde ses chiens ou ses béliers faire chacun leur travail de guidage et sert lui-même de point de repère pour tous les autres. La bataille homérique est aussi le lieu d'un tel ordre décentré et en permanence recentré sur son centre de contrôle, de la même manière que le troupeau se dissémine en sous-groupes guidés par les chiens ou les animaux dominants.

Les dieux, bergers du troupeau humain, garantissent dès lors que des loups ne se sont pas glissés dans la bergerie, c'est-à-dire des natures incurables qui tenteraient de soudoyer leurs gardiens pour commettre leurs forfaits en toute impunité. Ils luttent contre cette maladie naturelle de l'État que nous avons déjà évoquée, la forme civile de la pléonexie, l'injustice (906c4-6), et sont donc appelés à extraire du corps social ceux qui y sont devenus des loups au gré du devenir moral précédemment décrit. Ce texte étend donc lui aussi le pouvoir de purification des bergers au-delà de la période de fondation, en accord avec la leçon du Politique. Platon replace l'œuvre séparatrice des bergers homériques au sein de l'art politique.

Il y a un trait qui semblerait tout de même permettre au berger grec de ressembler au berger oriental de Foucault : la dimension individualisante de ce pouvoir. Mais il faut là encore renoncer à une fausse ressemblance. La réfutation de l'impiété dans le passage du livre $\mathrm{X}$ des Lois repose certes sur l'idée que les dieux ne négligent aucun détail et surveillent absolument tous les individus - personne ne peut espérer y échapper. Mais le pasteur n'est pourtant pas présent derrière chacun pour accompagner son progrès moral et le stimuler. Il y a pour cela des éducateurs : les dieux qui paissent les hommes se contentent d'entériner le devenir moral de chaque âme par un déplacement corrélatif qui en tire les conséquences ${ }^{52}$. Il s'agit d'un pouvoir de sanction, indifférent au fait que chacun en particulier soit devenu meilleur ou plus mauvais, mais intéressé à ce que la juste sanction et la juste récompense contribue au meilleur ordre global. Ce traitement sans bienveillance ni malveillance à l'égard des individus n'est jamais détaché de la perspective d'une justice globale : le tout n'a pas été disposé en vue de l'individu, c'est l'individu qui doit se soumettre à l'ordre du tout (Lois, X, 903b-c). La brebis n'est pas individualisée : elle est l'élément d'une multitude - elle est 
une singularité dans une masse dont le berger préserve globalement le salut physique et moral. La capacité d'isoler une singularité dans les multiplicités les plus inextricables est précisément encore tirée des analogies homériques et de leur transposition cosmologique, en particulier chez Anaxagore : le chef homérique, comme l'intelligence cosmique d'Anaxagore, saisit toute singularité dans la multitude ${ }^{53}$.

\section{L'ultime opération du pasteur politique : division du corps social et répartition des parts}

L'art des pasteurs homériques ne s'arrête pas là. Pour comprendre à quel point Platon en exploite les prérogatives, il faut revenir au début du livre V et sur le cas de la purification de la maladie de la cité introduite par la présence d'une masse de citoyens pauvres. La purification avait pour but d'éviter que se répande un problème insurmontable, sur lequel l'Étranger s'arrête.

Nous ne dissimulerons pas que nous avons de la chance - cette chance qui, nous l'avons dit, a favorisé la migration des Héraclides -, celle d'avoir échappé aux disputes redoutables et périlleuses que suscitent la propriété foncière, la remise des dettes et le partage des terres. Quand elle se trouve contrainte de légiférer en de telles matières, une cité ancienne ne peut ni laisser les choses en l'état ni non plus les rénover d'aucune façon : il ne lui reste pour ainsi dire que la ressource du souhait pieux et du changement léger et précautionneux, sur une longue période de temps. Ce souhait pieux, c'est qu'il se trouve toujours des rénovateurs eux-mêmes pourvus de terres considérables, pourvus aussi de débiteurs nombreux et souhaitant dans un esprit de conciliation partager une part de leurs biens avec ceux qui sont d'une certaine façon dans la nécessité, tantôt en remettant des dettes, tantôt en partageant des terres; des rénovateurs attachés en quelque sorte à la mesure et persuadés que l'appauvrissement réside moins dans une diminution de leur richesse que dans un accroissement de leurs convoitises. C'est là le principe le plus important pour la sauvegarde de la cité, et c'est là-dessus que, comme sur une base sûre, peut s'appuyer quiconque plus tard édifiera un ordre politique accordé à de pareilles circonstances. Mais si ce fondement est ruiné, il ne saurait y avoir par la suite de vie politique facile pour aucune cité. À cette difficulté, avons-nous déclaré, nous échappons. Néanmoins il serait plus correct, si nous n'y avions point échappé, de dire comment nous aurions bien pu nous y soustraire. Disons donc maintenant que c'est en associant le désintéressement à la justice; hormis ce moyen-là, il n'y a aucune autre issue, ni large ni étroite, pour s'en sortir. Bref, il faut poser cela comme un étai pour la cité. Car il faut s'arranger d'une manière ou d'une autre pour que les possessions ne suscitent pas de griefs entre leurs détenteurs. Sinon, quiconque a tant soit peu de bon sens ne consentira pas à être le premier à poursuivre des réformes aussi longtemps qu'il y a entre les 
bénéficiaires de celles-ci d'antiques sujets de querelle. Mais pour ceux à qui un dieu a offert, comme à nous en ce moment, de fonder une nouvelle cité où il n'existe pas encore d'inimitiés réciproques, ce serait joindre au comble de la malice un manque d'intelligence indigne d'un homme que de se rendre eux-mêmes responsables de ces inimitiés à l'occasion du partage des terres et des lieux de résidence ${ }^{54}$.

Voici donc un développement qui prend la mesure de cette seconde maladie de la cité que le pasteur avait pour but d'éviter en accomplissant la seconde forme de purification, la forme douce qui consiste à envoyer la partie pauvre de la population fonder une nouvelle colonie. Les problèmes que fait surgir dans une cité la perception d'un différentiel de richesse sont une maladie incurable de la cité. Une cité ancienne, affectée de cette difficulté, ne peut que difficilement s'en sortir : elle est confrontée à l'épineux problème de redistribuer des richesses déjà distribuées en l'absence de réserves prévues à cet effet - le problème qui est le prélude à la dispute entre Achille et Agamemnon au chant I de l'Iliade $e^{55}$. La seule solution est qu'elle dispose de dirigeants suffisamment fortunés pour être en capacité de distribuer des richesses ou des terres ou de remettre des dettes, et que cela permette de donner du temps à un corps malade. Si les interlocuteurs des Lois peuvent estimer être dans une autre situation, à condition d'agir avec intelligence, c'est qu'ils ont l'occasion de régler la répartition des terres. C'est sur celle-ci qu' ils enchaînent alors, avant de revenir à la description des magistratures, de leur mode de désignation et de leurs conditions d'accès, qui font précisément l'objet du « tissage » comme cela a été annoncé. On verra du reste que conformément aux préconisations du Politique, ce tissage sera appuyé sur des formes d'élection qui proposent de véritables concours de vertu, parachèvement de l'éducation qu'auront reçue les citoyens.

La répartition des lots de terrain en parts égales réparties entre égaux par tirage au sort est au fondement de la cité des Magnètes ${ }^{56}$. La répartition de la population en cinq mille quarante divisions est faite pour permettre à chaque foyer de recevoir une part égale de la terre, divisée en cinq mille quarante lots $(\mathrm{V}, 737 \mathrm{e})$. On remarque que ce nombre a l'avantage de pouvoir se diviser sans reste en deux, trois, quatre, cinq, six, sept, huit, neuf et $\mathrm{dix}^{57}$. Or l'Étranger souligne qu'un tel nombre est très utile pour la guerre comme pour un certain nombre de circonstances dans la paix, lorsque l'on veut répartir des charges ou des avantages : le nombre choisi se divise aussi bien en groupes de quatre (les classes - mille deux cent soixante foyers dans chaque groupe si elles étaient en

\section{Lois, V, 736c5-b9.}

55. I, 124-126; cf. Macé 2014.

56. Pradeau 2000.

57. Lois, V, 738a. Platon ne sait pas que cinq mille quarante est le factoriel de sept, ce qui explique sa faculté d'être aisément divisé par tous les nombres jusqu'à sept, ainsi que plusieurs multiples de ceux-ci. Mais il sait assurément que le nombre 5040 est riche en diviseurs. 
nombre égal) qu'en groupes de douze (les tribus, groupes de quatre cent vingt foyers). Il se prête à des recompositions : trente douzaines d'élus au conseil, soit trois cent soixante, cela correspond à une division de cinq mille quarante en quatorze, et on peut convertir ce chiffre en le divisant par classes, comme le fait l'Athénien pour mettre en place l'élection du conseil (on désignera quatre fois quatre-vingt-dix conseillers dans chaque classe). Or, comme on l'a remarqué, cette façon de tenir son groupe comme un nombre divisible de plusieurs façons, afin d'en faire le récipiendaire de biens et de missions, est un trait tiré de l'art des rangeurs d'hommes homériques ${ }^{58}$. On s'en rend compte en prêtant attention au type de mathématiques que manient aussi les chefs militaires homériques. Les « rangeurs d'hommes » et les pasteurs utilisent toutes sortes de critères de division : l'origine (les Rhodiens selon leurs trois

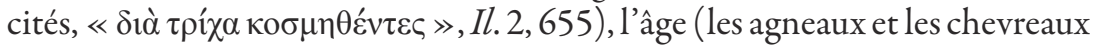
dans l'antre du cyclope ${ }^{59}$ ), les dispositions et l'équipement (chars / gens à pied par exemple $e^{60}$; ils divisent en différents nombres - ainsi faut-il savoir diviser son groupe en trois comme Ulysse en arrivant chez les Cyclopes,

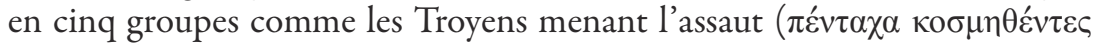
Il. 12, 87), en multitude de groupes de dix, comme l'armée achéenne divisée de manière imaginaire par Agamemnon en son premier discours (Il. 2, 124). Or ces divisions et recompositions éventuelles servent à distribuer nourriture et tâches au troupeau / à la troupe. Ainsi fait Ulysse, qui, arrivant sur l'île des Cyclopes $^{61}$, fait trois groupes à partir de douze bateaux pour aller à la chasse, avant de considérer de nouveau son groupe comme un ensemble de douze équipes au moment de répartir le nombre de chèvres issues de la chasse en parts égales ${ }^{62}$. Le pasteur de la cité n'est donc pas qu'un purificateur : son art de la séparation l'amène à savoir comment diviser le groupe dont il a la charge pour lui distribuer les biens et les missions, de telle sorte que l'égalité de la distribution garantisse la justice qui assure au corps social sa santé durable. Il a bien une tâche fondatrice, qui doit céder la place à l'ordinaire du travail politique. Mais on se souviendra de lui à chaque fois que l'on appelle une division du corps social pour lui assigner une tâche et s'affirmer comme partie prenante du politique.

58. Macé (à paraître-b).

59. En Od. 9, 220-222, Ulysse découvre dans l'antre du Cyclope Polyphème des stalles

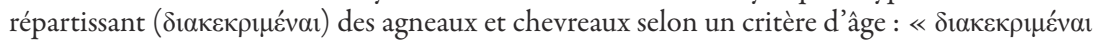

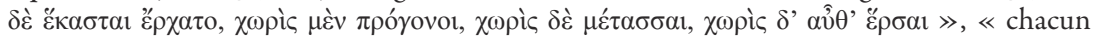
avait été enclos séparément, d'un côté les aînés, d'un autre les cadets, et, d'un autre encore, les nouveaux-nés ».

60. C'était la suggestion de Nestor. Peut-être faut-il penser qu'Ulysse, lorsqu'il déploie ses

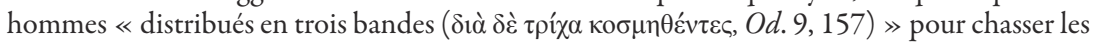
chèvres au pays des Cyclopes, utilise aussi un critère de spécialisation.

61. Homère, Od. IX, 157-160.

62. Macé 2014 p. 671-672. 
Platon, lecteur d'Homère, établit la présence d'un pastorat au fondement de la cité, d'une manière qui confère à son art la responsabilité de la sauvegarde de celle-ci dans le temps, de son égalité réelle et perçue, et de sa capacité d'action. Apparaît donc une espèce du pastorat dont les analyses de Michel Foucault ont manqué l'embranchement : non pas le pastorat bienveillant qui vise à sauver chaque brebis, mais celui qui sauve le groupe en le purifiant des brebis galeuses; non pas celui qui se rend présent derrière chacune pour l'inviter au progrès moral, mais celle qui prend acte du devenir moral de chacune en vue de sa prochaine affectation ; art des fondations et des purifications salutaires plutôt qu'art du contrôle permanent ; art des multiplicités et des singularités plutôt qu'art des individualités ; art de la répartition des masses, des biens, art de l'égalité réelle des distributions, art de la force des collectifs, de leur justice et de leur courage.

En quoi ce portrait du pasteur platonicien modifie-t-il l'image que nous pouvons nous faire du pastorat grec ? Tout d'abord il n'est pas sûr qu' il faille chercher des spécificités culturelles absolues, distinguant essentiellement le pastorat des uns de ceux des autres, en particulier celui qui relèverait des « indo-européens ». On a du reste pu défendre l'idée que le paradigme du pastorat en matière de pensée politique distinguait fortement les sociétés méditerranéennes de celles d'Asie, privilégiant quant à elle les analogies tirées du modèle de l'agriculteur qui accompagne la pousse des végétaux ${ }^{63}$. Comme j'ai pu le suggérer ailleurs, le rapport spécifique des Grecs à la nature, que l'on a aussi parfois voulu expliquer par un trait indo-européen, tient plutôt à leur manière de mêler les analogies qu'ils tirent de leurs pratiques pastorales méditerranéennes avec les schèmes de la pousse végétale favorisés par les cultures d'Orient ${ }^{64}$. Les singularité culturelles sont souvent des combinaisons originales de traits communs, et chaque auteur au sein d'une culture donnée est susceptible d'altérer la combinaison majoritaire dans son environnement culturel.

Aussi pourrait-on dire que la bienveillance elle-même, celle que Foucault réservait au pastorat hébraïque ou au Moyen-Orient en général, se trouve intégrée et modifiée chez nos auteurs grecs, du fait de la prépondérance des pratiques militaires dans le développement de l'analogie pastorale en Grèce ancienne ${ }^{65}$. Etre au service de son troupeau est un trait qui semble particulièrement mis en avant par les écrivains socratiques, comme le Platon de la République ou le Xénophon de la Cyropédie. La bienveillance promise à chaque individu se mue ici en soin du groupe, en souci de son bonheur collectif et global : Xénophon compare Cyrus à un berger lorsqu'il veut

63. Haudricourt 1962.

64. Macé 2017, en particulier p. 211-215.

65. Sur cette prépondérance, voir les références déjà mentionnées en introduction, notamment Brock 2013 p. 43. 
souligner la $\theta \varepsilon \rho \alpha \pi \varepsilon i ́ \alpha$ que celui-ci déploie à l'endroit de ses sujets en cherchant à leur procurer le bonheur dont ils sont capables, car c'est en étant heureux qu'ils le servent mieux (Cyropédie, VIII, 2, 13). Platon insiste sur le fait que Cyrus, en donnant à chacun la confiance de s'avancer pour livrer au roi un conseil avisé, favorise l'aptitude de chacun à nourrir l'intelligence collective, la « communauté de pensée » (Lois, III, 694b 1-6). Le pastorat est identifié comme art de production des collectifs vertueux, où chacun s'épanouit par le fait de rendre le tout plus robuste.

Il reste à mesurer ce que la prise en compte du pastorat politique grec change à l'histoire de la gouvernementalité. On pourrait craindre qu'elle fasse perdre à celle-ci le recours que lui offrait l'histoire des pratiques grecques : cet homme grec qui, selon Foucault, n'avait jamais consenti à se laisser « considérer comme une brebis parmi les brebis ${ }^{66}$, semblaient offrir aux individus assujettis par le déploiement de la bienveillance envahissante du pastorat chrétien, la résistance des techniques de soi $^{67}$. Le fait qu'un pastorat surgisse sur le terrain grec, susceptible de réclamer la souveraineté sur toutes les techniques de soi individuelles, de réclamer le droit de purifier le corps social de ceux qu'aucune technique de soi n'aura pu transformer et d'affecter à sa guise ceux qu' il juge bons pour le service, pourrait rendre l'option antique moins désirable à l'individu moderne, et surtout jeter un doute sur la capacité des techniques grecques d'offrir une vision émancipée de la subjectivation. Les traits structurels du pastorat grec pourraient néanmoins expliquer que les individus qu'il produit aient pu sembler, à un regard sensible aux effets de l'autre pastorat, libérés de celui-ci. Être brebis se dit peut-être en différents sens : l'être comme membre d'un collectif agissant, soucieux de l'égalité de sa part reçue, en biens comme en missions, semble différent de l'être comme sujet assigné à la tâche de rendre compte intimement de son devenir moral. Il faudrait donc regarder quels effets la restitution de cet autre pastorat pourrait bien avoir au sein d'une histoire de la gouvernementalité comme au sein d'une histoire des pratiques de subjectivation. On pourrait s'attendre à ce qu'elle trace dans notre modernité les contours d'une histoire différente, attentive à d'autres institutions, à d'autres savoirs, à d'autres révolutions et à d'autres luttes. 


\section{BIBLIOGRAPHIE}

Allen, T. W. 2000 (éd.) : Homeri Ilias, 3 vol., Oxford, 2000 [1931].

Azoulay, V. 2004 : Xénophon et les grâces du pouvoir : de la charis au charisme, Paris, 2004 (Histoire ancienne et médiévale, 77).

- 2014 : « Repenser le politique en Grèce ancienne », Annales. Histoire, Sciences Sociales, $69^{\mathrm{e}}$ année/3 (2014), p. 605-626.

Benveniste, É. 1969 : Le vocabulaire des institutions indo-européennes. 2, Pouvoir, droit, religion, Paris, 1969.

Blondell, R. 2005 : « From Fleece to Fabric : Weaving Culture in Plato's Statesman », Oxford Studies in Ancient Philosophy, 28 (2005), p. 23-75.

Brisson, L. \& J.-F. Pradeau 2008 (trad.) : Platon, Lois, dans L. Brisson (dir.), Platon, CEuvres complètes, Paris, 2008, p. 679-1008.

- 2008 (trad.) : Platon, Le Politique, dans L. Brisson (dir.), Platon, CEuvres complètes, Paris, 2008, p. 1367-1434.

Brock, R. 2013 : Greek Political Imagery from Homer to Aristotle, London, 2013.

El Murr, D. 2002 : « La symplokè politikè : le paradigme du tissage dans le Politique de Platon, ou les raisons d'un paradigme "arbitraire" », Kairos, 19 (2002), p. 49-95.

- 2013 : «Adieu au pasteur ? Remarques sur le pastorat politique dans le Politique de Platon », dans A. Castel-Bouchouchi, M. Dixsaut et G. Kevorkian (éd.), Platon, Paris, 2013 (Lectures de...), p. 171-184.

- 2014 : Savoir et gouverner : essai sur la science politique platonicienne, Paris, 2014

(Tradition de la pensée classique).

Foucault, M. 1981 : « "Omnes et singulatim” : Towards a Criticism of Political Reason », dans S. M. McMurrin (éd.), The Tanner Lectures on Human Values, 2 (1981), p. 223-254 (URL : http://tannerlectures.utah.edu/_documents/ato-z/f/foucault81.pdf).

- 1986 : « "Omnes et singulatim" : vers une critique de la raison politique », Le Débat 41/4 (1986), p. 5-36.

- 1994 : Dits et écrits, 1954-1988 : vol. 4, 1980-1988, éd. établie sous la dir. de Daniel Defert et François Ewald, avec la collab. de Jacques Lagrange, Paris, 1994 (Bibliothèque des sciences humaines).

- 2001 : L'Herméneutique du sujet: cours au Collège de France, 1981-1982, Paris, 2001 (Hautes études).

- 2004 : Sécurité, territoire, population: cours au Collège de France, 1977-1978, Paris, 2004 (Hautes études).

Haubold, J. 2000 : Homer's People: Epic Poetry and Social Formation, Cambridge, 2000 (Cambridge Classical Studies).

Haudricourt, A. G. 1962 : « Domestication des animaux, culture des plantes et traitement d'autrui », L'Homme, $2 / 1$ (1962), p. 40-50.

Joly, H. 1994 : Le Renversement platonicien : logos, épistémè, polis, seconde éd. corr., Paris, 1994 (Tradition de la pensée classique). 
MACÉ, A. 2010 : «Santé des corps, des esprits, des cités : un modèle antique de liaison entre pathologie sociale et pathologie psychique », dans S. Haber (dir.), Des Pathologies Sociales Aux Pathologies Mentales, Besançon, 2010 (Annales littéraires de l'Université de Franche-Comté, 867), p. 31-54.

- 2014 : «Deux formes du commun en Grèce ancienne », Annales. Histoire, Sciences Sociales 69 année/3 (2014), p. 659-688.

- 2017 : « Nature among the Greeks : Empirical Philology and the Ontological Turn in Historical Anthropology », dans P. Charbonnier, G. Salmon \& P. Skafish (éd.), Comparative Metaphysics, Ontology after Anthropology, London / New-York, 2017, p. 201-220.

— à paraître-a : «La justice cosmique dans les Lois : Platon lecteur d'Homère et d'Anaxagore », dans A. Jaulin et al. (éd.), Philia/Dikè : aspects du lien social et politique en Grèce ancienne, Paris (à paraître-a).

— à paraître-b : « Platon, le tirage au sort au fondement de la communauté politique », dans Y. Syntomer \& L. Rabatel (éd.), Tirage au sort et démocratie, (à paraître-b).

Macé, A. \& A.-L. Therme 2013 : « Anaxagore et Homère : trier les moutons, trier les hommes, trier l'univers », dans M.-L. Desclos \& F. Fronterotta (dir.), La Sagesse présocratique : communication des savoirs en Grèce archä̈que : des lieux et des hommes, Paris, 2013 (Recherches), p. 235-261.

Narcy, M. 1995 : « La critique de Socrate par l'Étranger dans le Politique », dans C. J. Rowe (éd.), Reading the Statesman: Proceedings of the III Symposium Platonicum, Sankt Augustin, 1995 (International Plato studies, 4), p. 227-235.

Petit, A. 1999 : « Le pastorat ou l'impossible raccourci théologico-politique », dans E. Cattin, L. Jaffro \& A. Petit (éd.), Figures du théologico-politique, Paris, 1999 (Problèmes et controverses), p. 9-24.

Pontier, P. 2006 : Trouble et ordre chez Platon et Xénophon, Paris, 2006 (Histoire des doctrines de l'Antiquité classique, 34).

PradeaU, J.-F. 2000 : «L'économie politique des Lois : remarques sur l'institution des KLHROI », Cabiers du Centre Gustave Glotz, 11/1 (2000), p. 25-36.

Skemp, J. B. 1952 (trad.) : Plato, Plato's Statesman : a translation of the Politicus, with introd. essays \& footnotes, London / New Haven, 1952 (Rare Masterpieces of Philosophy and Science).

Therme, A.-L. \& A. Macé 2016 : « L’immanence de la puissance infinie : le vôิৎ d'Anaxagore à la lumière d'Homère », Methodos. Savoirs et textes, [En ligne] 16 (2016), URL : http://methodos.revues.org/4477 ; DOI : 10.4000/ methodos.4477. 